\title{
Base-free, Vanadium-catalyzed Conversion of Chitin into Acetic Acid under Low Oxygen Pressure
}

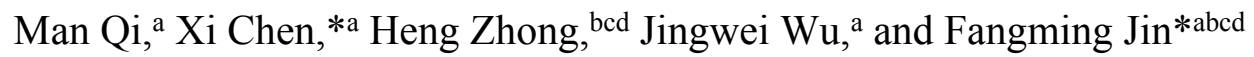

${ }^{a}$ China-UK Low Carbon College, Shanghai Jiao Tong University, 3 Yinlian Rd, 201306, Shanghai, China.

${ }^{b}$ School of Environmental Science and Engineering, State Key Lab of Metal Matrix Composites, Shanghai Jiao Tong University, No. 800, Dongchuan Road, Shanghai 200240, China.

${ }^{c}$ Center of Hydrogen Science, Shanghai Jiao Tong University, No. 800, Dongchuan Road, Shanghai, 200240, China

${ }^{d}$ Shanghai Institute of Pollution Control and Ecological Security, Shanghai, 200092, China

*Corresponding emails: chenxi-1cc@sjtu.edu.cn;

fmjin@sjtu.edu.cn.

\section{Table of Contents}

Number of pages in the supporting information: 8

Number of figures in the supporting information: 5

Number of tables in the supporting information: 4 
Table S1. The production of AA using $\mathrm{CuO}$ catalyst in various acidic/basic solutions

\begin{tabular}{|c|c|c|c|c|}
\hline Entry & Catalyst & $\mathrm{c}(\mathrm{HCl})$ & c $(\mathrm{NaOH})$ & AA yield $(\%)$ \\
\hline 1 & $\mathrm{CuO}$ & $0.05 \mathrm{M}$ & l & 23.1 \\
\hline 2 & $\mathrm{CuO}$ & $0.1 \mathrm{M}$ & / & 23.9 \\
\hline 3 & $\mathrm{CuO}$ & $0.2 \mathrm{M}$ & l & 23.6 \\
\hline 4 & / & $0.1 \mathrm{M}$ & / & 23.0 \\
\hline 5 & $\mathrm{CuO}$ & l & $0.05 \mathrm{M}$ & 23.0 \\
\hline 6 & $\mathrm{CuO}$ & / & $0.1 \mathrm{M}$ & 29.3 \\
\hline 7 & $\mathrm{CuO}$ & l & $0.2 \mathrm{M}$ & 32.0 \\
\hline 8 & / & / & $0.1 \mathrm{M}$ & 23.4 \\
\hline
\end{tabular}

Reaction conditions: $30 \mathrm{mg} \mathrm{NAG}$, catalyst to substrate ratio $(\mathrm{mol} / \mathrm{mol})=1 / 3,0.5 \mathrm{MPa}$ $\mathrm{O}_{2}$ gas, $3 \mathrm{~mL}$ water, $220{ }^{\circ} \mathrm{C}, 2 \mathrm{~h}$. 


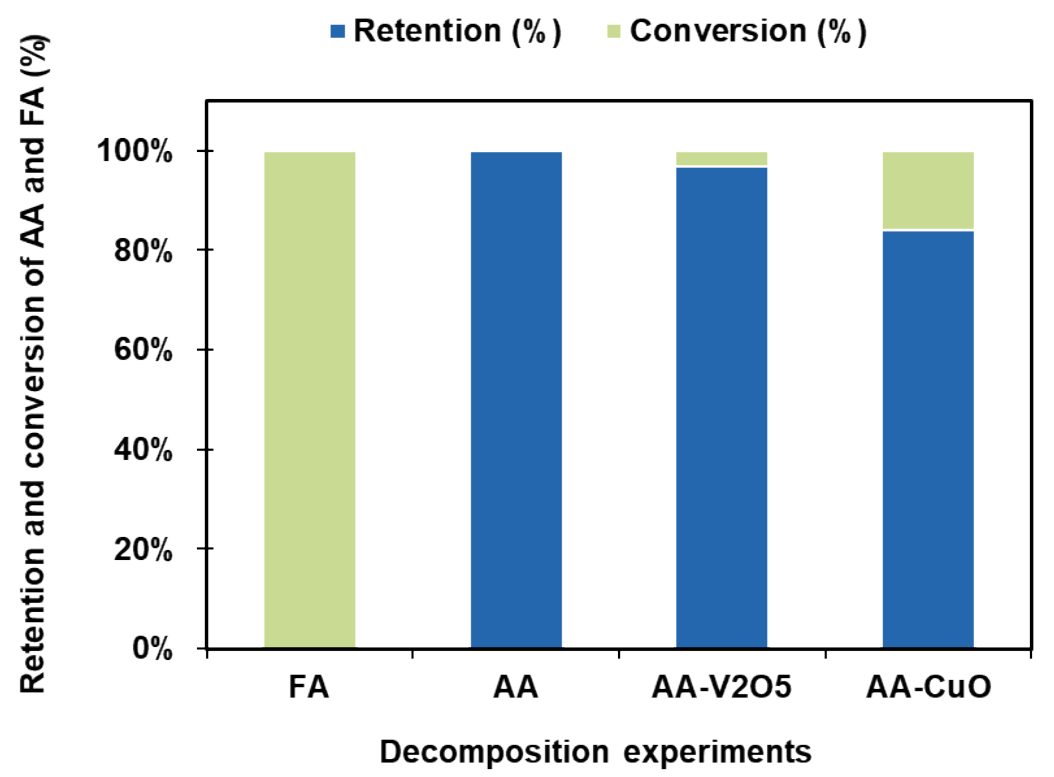

Figure S1. The decomposition tests for FA and AA in the absence and presence of the catalyst. Reaction conditions: $30 \mathrm{mg} \mathrm{NAG}$, catalyst to substrate ratio $(\mathrm{mol} / \mathrm{mol})=1 / 3$, $0.5 \mathrm{MPa} \mathrm{O}_{2}$ gas, $3 \mathrm{~mL}$ water, $220^{\circ} \mathrm{C}, 2 \mathrm{~h}$. 


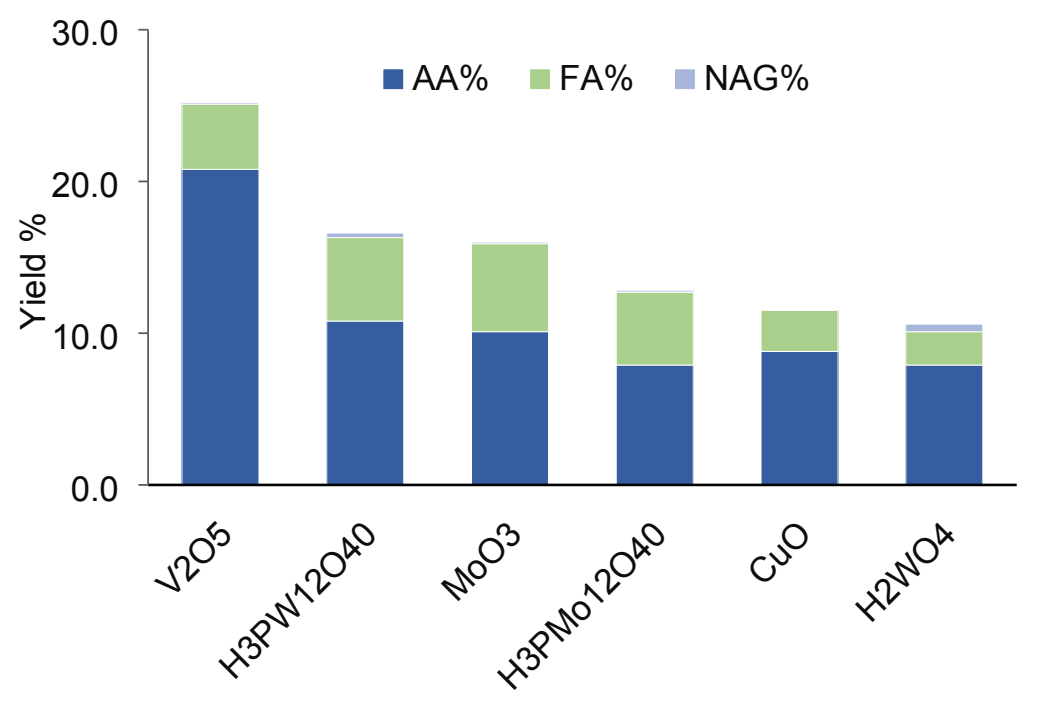

Figure S2. The influences of different catalysts on AA yield from chitin. Reaction conditions: $27 \mathrm{mg}$ chitin, catalyst to substrate ratio $(\mathrm{mol} / \mathrm{mol})=1 / 3,0.5 \mathrm{MPa} \mathrm{O}_{2}$ gas, 3 $\mathrm{mL}$ water, at $220^{\circ} \mathrm{C}, 2 \mathrm{~h}$.

Table S2. Chitin conversion into AA using $\mathrm{MoO}_{3}$ as the catalyst

\begin{tabular}{cccccc}
\hline Entry & Substrate & Temperature $\left({ }^{\circ} \mathrm{C}\right)$ & Time $(\mathrm{h})$ & Additive & AA yield (\%) \\
\hline 1 & Chitin & 220 & 2 & $/$ & 10.1 \\
2 & Chitin & 220 & 2.5 & $/$ & 10.3 \\
3 & Chitin & 220 & 3 & $/$ & 16.0 \\
4 & Chitin & 220 & 3 & $0.02 \mathrm{M} \mathrm{HCl}$ & 14.4 \\
5 & Chitin & 220 & 2 & $0.04 \mathrm{M} \mathrm{HCl}$ & 20.3 \\
\hline
\end{tabular}

Reaction conditions: $27 \mathrm{mg}$ chitin, $\mathrm{MoO}_{3}$ to chitin ratio $=1 / 3,0.5 \mathrm{MPa} \mathrm{O}, 3 \mathrm{~mL}$ water. 
Table S3. Efficiency of different extractants to extract AA

\begin{tabular}{|c|c|c|c|c|}
\hline Entry & Extractant & Times & Extracted solution & Efficiency ( \%) \\
\hline 1 & Dichloromethane & 1 & Acetic acid solution & 4.5 \\
\hline 2 & Ethyl acetate & 1 & Acetic acid solution & 14.7 \\
\hline 3 & n-Heptanol & 1 & Acetic acid solution & 43.3 \\
\hline 4 & Octanol & 1 & Acetic acid solution & 44.4 \\
\hline 5 & 2-Ethyl-1-hexanol & 1 & Acetic acid solution & 44.2 \\
\hline 6 & n-Heptanol & 4 & Acetic acid solution & 85.6 \\
\hline 7 & Octanol & 4 & Acetic acid solution & 80.3 \\
\hline 8 & n-Heptanol & 4 & $\begin{array}{l}\text { Aqueous solution of } \\
\text { chitin conversion }\end{array}$ & 62.5 \\
\hline 9 & n-Heptanol & 6 & $\begin{array}{l}\text { Aqueous solution of } \\
\text { chitin conversion }\end{array}$ & 68.2 \\
\hline
\end{tabular}

Reaction conditions: $25{ }^{\circ} \mathrm{C}, 1 \mathrm{~h}, 600 \mathrm{rpm}$. For 1-7, $2.5 \mathrm{~mL} 180 \mathrm{mmol} / \mathrm{L}$ AA standard solutions were extracted by $2.5 \mathrm{~mL}$ different extractants; for $8-9,2.5 \mathrm{~mL}$ solution after chitin reaction was extracted by $2.5 \mathrm{~mL}$-heptanol. 


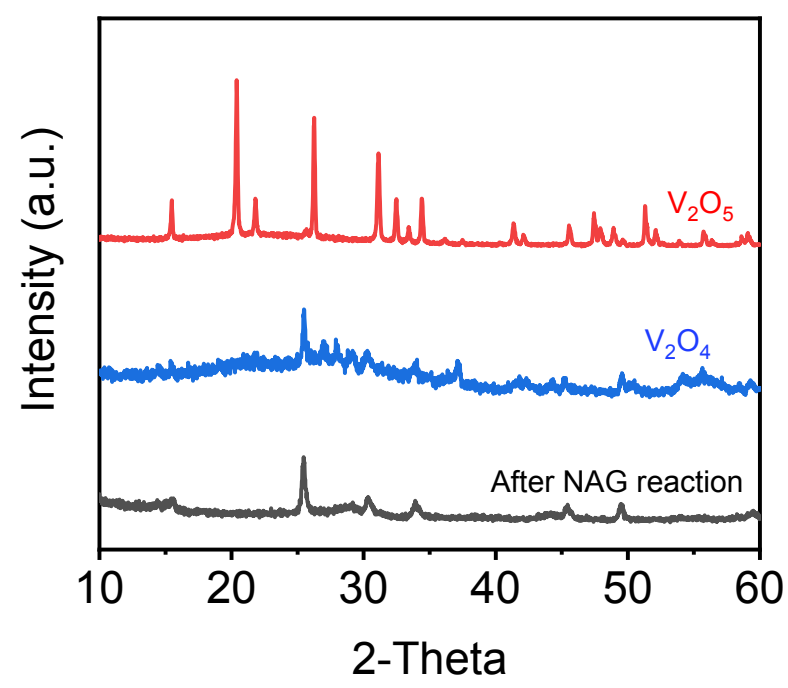

Figure S3. XRD of the solid residue after NAG conversion. Reaction conditions: 30 mg NAG, catalyst to substrate ratio $(\mathrm{mol} / \mathrm{mol})=1 / 3,0.5 \mathrm{MPa} \mathrm{O}_{2}$ gas, $3 \mathrm{~mL}$ water, $2 \mathrm{~h}$.

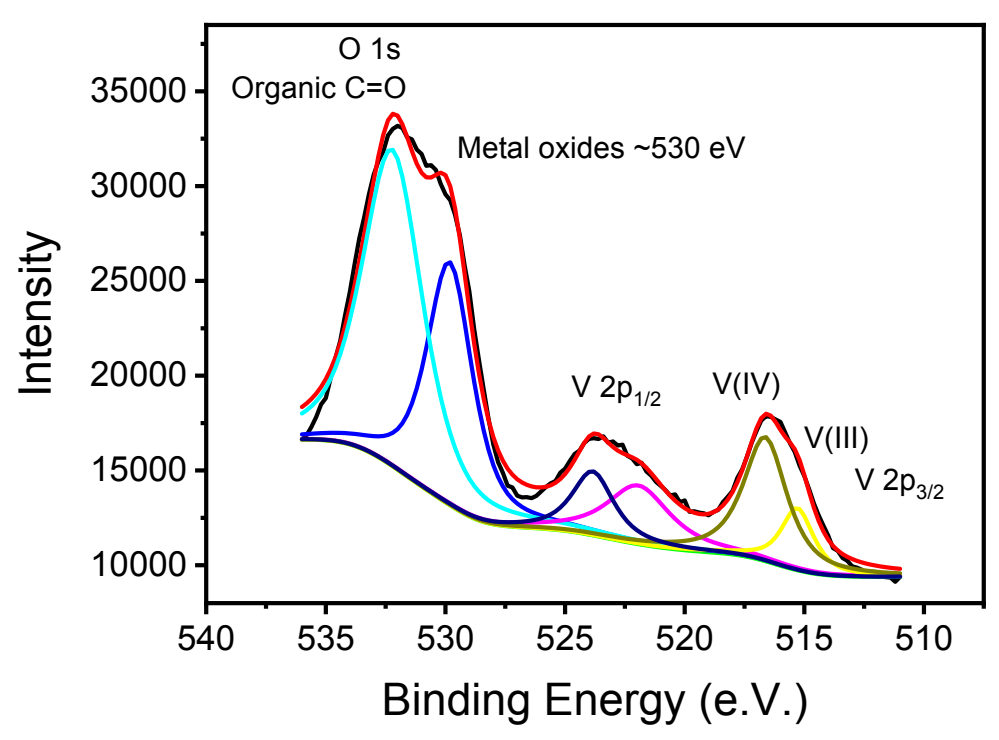

Figure S4. XPS of the solid residue after NAG conversion. Reaction conditions: $30 \mathrm{mg}$ NAG, catalyst to substrate ratio $(\mathrm{mol} / \mathrm{mol})=1 / 3,0.5 \mathrm{MPa} \mathrm{O}_{2}$ gas, $3 \mathrm{~mL}$ water, for $2 \mathrm{~h}$.

Table S4. AA production using several homogeneous vanadium salts 


\begin{tabular}{ccc}
\hline Entry & Catalyst & AA yield (\%) \\
\hline 1 & $\mathrm{Na}_{3} \mathrm{VO}_{4}$ & 21.8 \\
2 & $\mathrm{NH}_{4} \mathrm{VO}_{3}$ & 20.8 \\
3 & $\mathrm{VOSO}_{4}$ & 24.8 \\
\hline
\end{tabular}

Reaction conditions: $30 \mathrm{mg}$ NAG as the substrate, catalyst to substrate ratio ( $\mathrm{mol} / \mathrm{mol})$

$=1 / 3,0.5 \mathrm{MPa} \mathrm{O}_{2}$ gas, $3 \mathrm{~mL}$ water, at $220^{\circ} \mathrm{C}, 2 \mathrm{~h}$. 


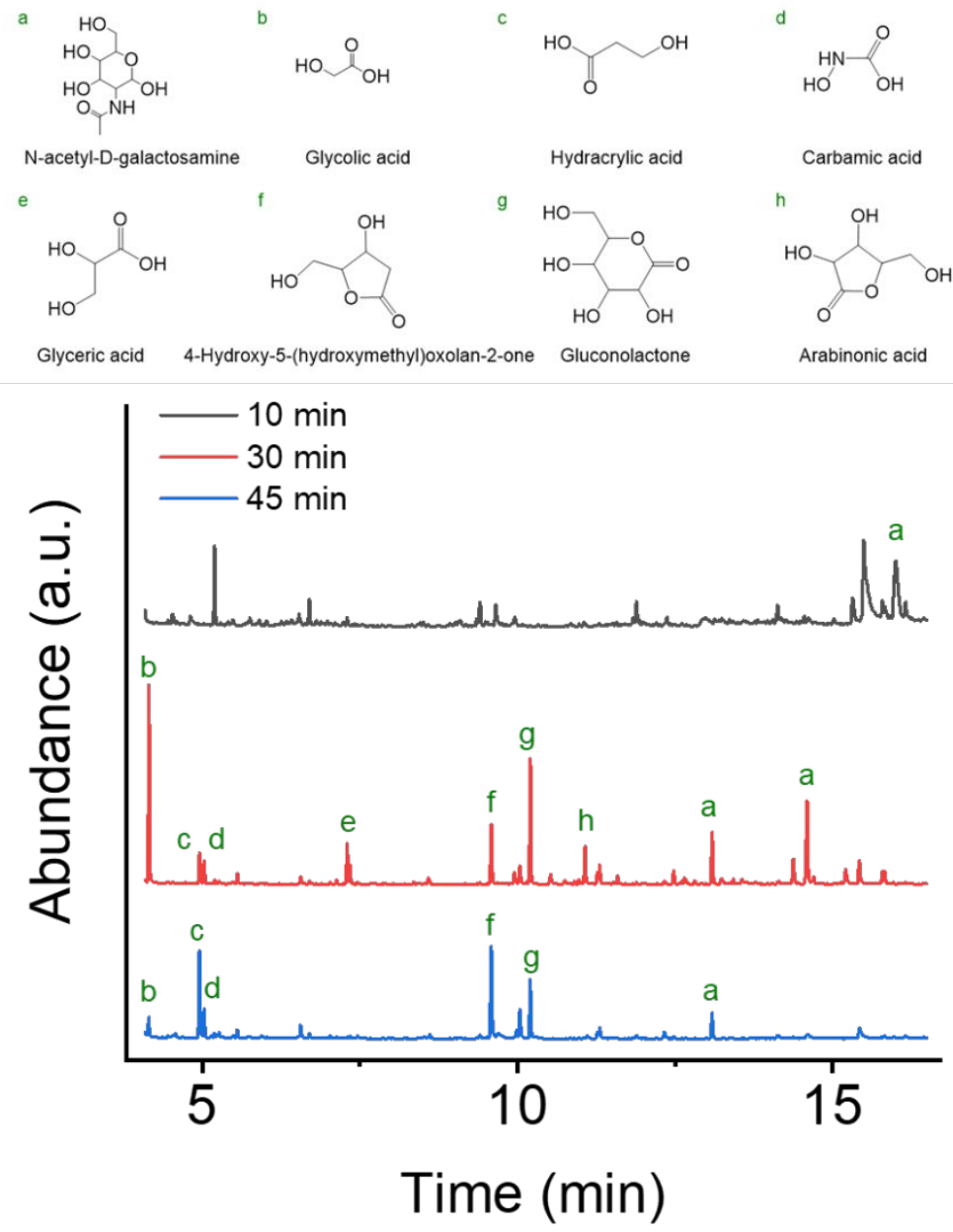

Figure S5. The GC-MS spectra of silylated products after NAG conversion. Reaction conditions: $30 \mathrm{mg}$ NAG, catalyst to substrate ratio $(\mathrm{mol} / \mathrm{mol})=1 / 3,0.5 \mathrm{MPa} \mathrm{O}_{2}$ gas, 3 $\mathrm{mL}$ water, for $10 \mathrm{~min}, 30 \mathrm{~min}$, and $45 \mathrm{~min}$. 